\title{
Pleural Tuberculosis in A 30 Year Old Male Misdiagnosed as Pleural Neoplasia: A Case Report (The Role of Imaging in A Low Resource Setting)
}

\begin{abstract}
Danfulani Mohammed*, Haruna Gele Ibrahim, Shamsuddeen Aliyu and Sule Muhammad Baba Abstract: Tuberculosis (TB) is a leading cause of mortality worldwide with increasing mortality rate. The pleura is a common extra pulmonary site of involvement and it is the most common form of extra pulmonary TB only less frequent than lymph nodes involvement. All age groups can be affected by TB. Tuberculous involvement of the pleura usually presents as pleural effusion, empyema or pleural thickening. Rarely, it can present as a pleural mass with or without lung parenchymal involvement. This case is presented to highlight the role of imaging especially plain x-ray and computed tomography to resolve the confusion.

Article History

Received: 08.11 .2020

Accepted: 19.11 .2020

Published: 22.11 .2020

Journal homepage:

https://www.easpublisher.com

Keywords: Pleural Tuberculosis, Pleural Neoplasia, Imaging, Case Report.
\end{abstract}

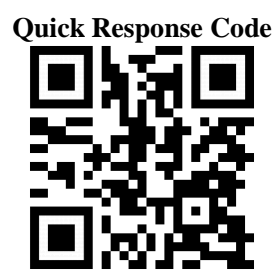

\begin{abstract}
Copyright (C) 2020 The Author(s): This is an open-access article distributed under the terms of the Creative Commons Attribution 4.0 International License (CC BY-NC 4.0) which permits unrestricted use, distribution, and reproduction in any medium for non-commercial use provided the original author and source are credited.
\end{abstract}

\section{INTRODUCTION}

Tuberculosis (TB) is a leading cause of mortality worldwide with increasing mortality rate [1]. The pleura is a common extra pulmonary site of involvement[1] and it is the most common form of extra pulmonary TB only less frequent than lymph nodes involvement [2]. All age groups can be affected by TB. Tuberculous involvement of the pleura usually presents as pleural effusion, empyema or pleural thickening. Rarely, it can present as a pleural mass with or without lung parenchymal involvement. The imaging features of pleural tuberculosis can be similar to those of malignant pleural disease [3]. Establishing pleural TB as a cause of pleural effusion or mass can sometimes be challenging [3].

This case of pleural TB in a 30 year old male misdiagnosed as pleural neoplasia is presented due to the diagnostic challenge encountered in its management.

\section{Case Report}

$\mathrm{NM}$ is a 30 year old male University student who presented to the pulmonology unit and later to cardio-thoracic unit of Usmanu Danfodio University Teaching Hospital Sokoto with two months history of cough and right sided chest pain. Cough was productive of scanty mucoid sputum but no haemoptysis. The chest pain was dull, non-radiating and worse on exertion. No fever, weight loss or drenching night sweat. No history of cigarette smoking, exposure to organic or inorganic dust or industrial chemicals. No past history of trauma. $\mathrm{He}$ is not a known human immunodeficiency virus (HIV) positive patient.

Physical examination showed a healthy looking man, afebrile, not pale, anicteric, and acyanosed. There were dull percussion notes over the right middle and lower lung zones with decrease air entry suggestive of fluid. Thoracocentesis yielded dry tap. Other systemic examinations were normal. A clinical assessment of suspected pulmonary tuberculosis was made based on history of chronic cough and physical examination findings.

Initial laboratory evaluation (sputum acid fast bacilli and Mantoux test) was negative for pulmonary TB. Sputum cytology was reported as suspicious of malignancy. The retroviral screening was negative. Initial chest radiograph showed a large homogenous opacity with well circumscribed medial margin in the right lateral aspect of the middle and lower lung zones. It showed a convex border medially and an obtuse angle with the lung parenchyma (fig. 1). There were patchy parenchymal opacities in the right middle lung zone. 
The heart, mediastinum and bony thorax were normal. The lateral radiograph showed the homogenous opacity projected over the heart shadow. An assessment of right pleural based mass was made and computed tomography (CT) of the chest was advised for further evaluation.

Chest CT showed a loculated pleural effusion $(H U=14)$ with contrast enhancement of the visceral pleura on the right postero-lateral chest wall. There was thickening of the pleura posteriorly. A small oval slightly enhancing isodense mass with spiculated margins was also seen attached to the pleura posteriorly (fig. 2 and 3). This mass had a central hyperdense focus $(\mathrm{HU}=106)$ ? Calcification. The bony thorax was essentially normal. A CT assessment of a pleural based mass, most probably neoplasia, with loculated malignant pleural effusion was made. Pulmonary TB was given as a differential and image guided thoracocentesis was suggested.

Repeat sputum cytology was reported as suspicious of malignancy. The patient later had thoracotomy with decortications and pleurectomy. The pleural specimen was sent for histology and it showed variable sized granulomata with central area of necrosis. Special stain was positive for pulmonary TB.

Post-operative period was uneventful. The post-operative chest radiograph showed clearance of the loculated pleural effusion. There were patchy opacities in the right lung fields presumably due to post-surgical pulmonary changes. Chest tube was noted in situ. $\mathrm{He}$ was placed on anti-TB drugs and discharged one week later. He remained asymptomatic on routine follow up for up to two months.

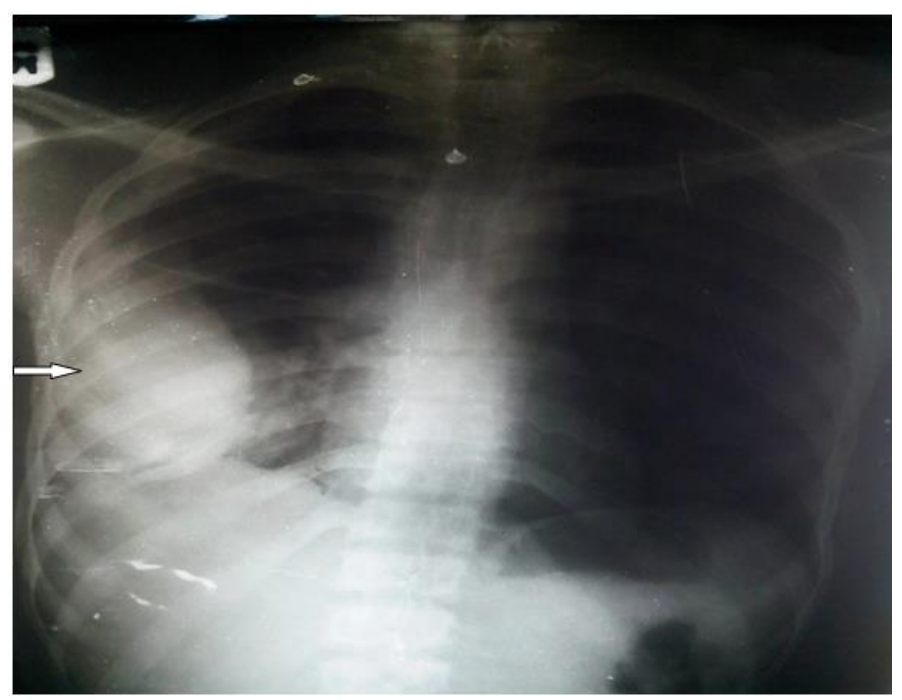

Fig-1: Chest radiograph postero-anterior view showing a homogenous opacity in the lateral aspect of the right hemithorax with convex border medially and making an obtuse angle to the lung parenchymal (arrow). The opacity is obliterating the lateral 2/3 of the right hemidiaphragm. Note the adjacent patchy parenchymal lung opacities as well

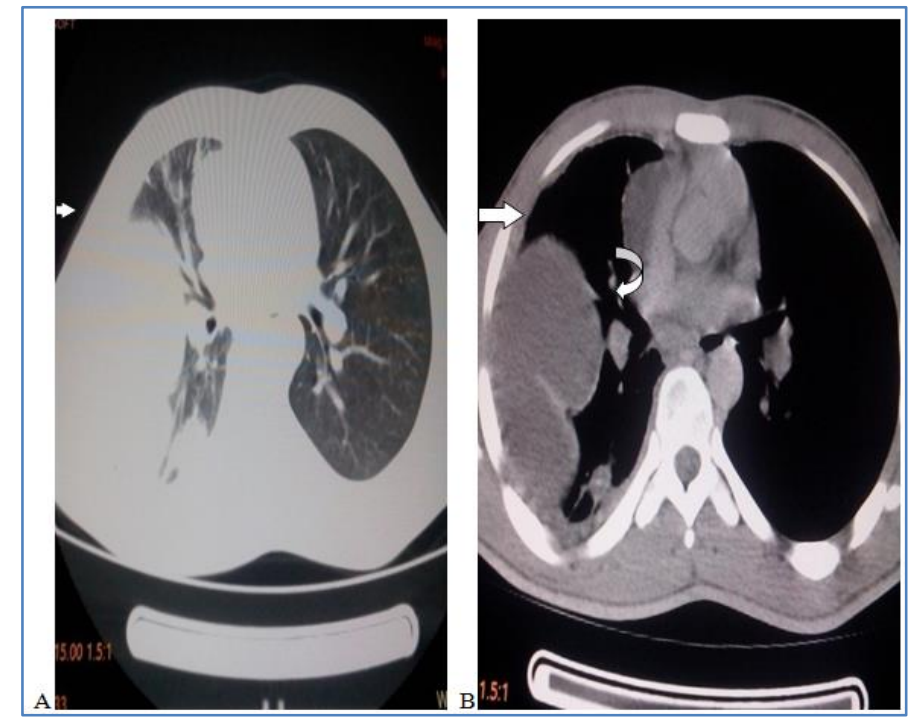

Fig-2: CT of the chest axial views, lung (A) and mediastinal (B) windows showing loculated pleural effusion (straight arrows). There is pleural thickening posteriorly with a nodule showing spiculated margin attached to the pleura in this region (curved arrow) 


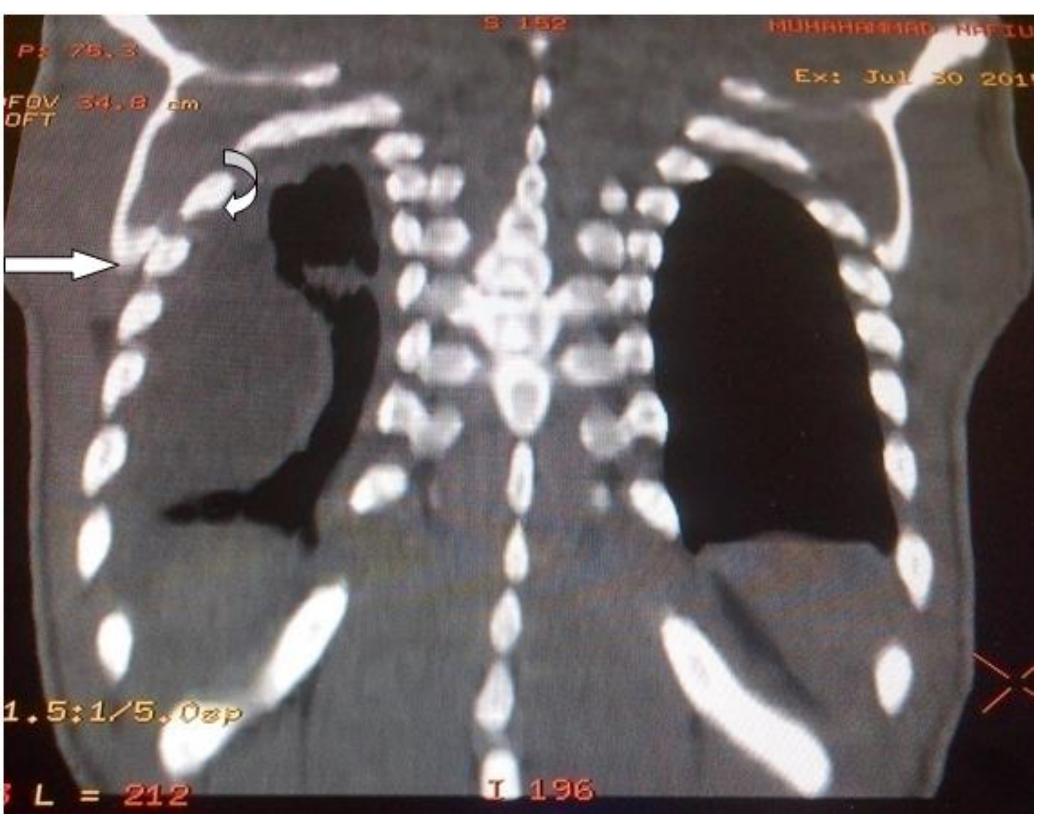

Fig-3: Chest CT coronal view, posterior lung region showing loculated pleural effusion on the lateral chest wall (straight arrow). Note a spiculated nodule attached to the pleura as well (curved arrow)

\section{DISCUSSION}

Tuberculosis (TB) is transmitted via air droplets nuclei that are produced when persons with pulmonary or laryngeal TB cough, sneeze, speak or sing [4]. Infection occurs when a susceptible person inhales the droplet nuclei. These then spread from the alveoli through the lymphatic channels to regional lymph nodes and blood stream to more distant sites in the body. Pleural TB infection is usually as a result of rupture of sub-pleural caseous focus within the lung into the pleural space. It may occasionally be as a result of haematogenous dissemination or contamination from adjacent infected lymph nodes [3, 4].

Pleural TB is the most frequently occurring form of extra pulmonary disease in adults [5]. In up to $40 \%$ of cases, the lung parenchyma is concomitantly involved [5]. In this case, an area of lung parenchymal opacities was seen adjacent to the loculated pleural effusion which was initially presumed to be due to inflammation caused by suspected pleural based mass.

Pulmonary TB classically presents with productive cough [4], a feature seen in the case here presented. Typical presentations of TB also include weight loss, low grade fever and drenching night sweats [4]. However these supportive features were not seen in this patient. In addition to above symptoms, pleural TB present with pleuritic chest pain [4] as in the case presented.

Pleural involvement in TB is reported to be associated with HIV [1, 6, 7]. In the index case however, the patient was HIV negative.

Pleural infection in TB is usually characterized by an effusion in early stages or by diffuse pleural thickening and adhesions, which may calcify in long standing cases. Pleural thickening is a non-specific finding and may result from other conditions such as haemothorax, empyema, and recurrent pneumothorax $[3,8]$. It may also occur in malignant pleural dissemination (MPD), however MPD is associated with more nodular pleural thickening than pleural TB particularly with nodules $>10 \mathrm{~mm}$ [8].

Pleural effusion can occur in any form of pulmonary tuberculosis. Neither the clinical features nor any of the imaging modalities are of much help in the diagnosis of a tuberculous pleura effusion. Coexisting parenchymal disease detected radiographically in about one third of the patients with an effusion serves as a marker of active pulmonary tuberculosis [9]. Computed tomography (CT) of the chest may show lymphadenopathy, pulmonary infiltrates or cavitations not seen on chest radiograph, which although nonspecific, may help to distinguish pleural TB from other causes like malignancy [9]. These other features were also absent in the chest CT of the case here presented.

The definitive diagnosis of TB as a cause of pleural fluid seen on imaging rests on microbiological and histological analysis of aspirated pleural fluid and the biopsied pleural tissue. Mycobacteria are seen in pleural fluid only in $10-20 \%$ of cases whereas a culture though positive in $25-50 \%$ of cases, takes a minimum of 6-8 weeks by conventional method to be of any clinical utility. Pleural biopsy will show granulomas in the parietal pleura in $50-80 \%$ of patients and if cultured will grow mycobacterium in $55-80 \%$ of cases [9].

Treatment with anti-tuberculous drugs is mainstay of management of extra pulmonary 
tuberculosis. Large volume of pleural fluid associated with respiratory difficulties will require surgical drainage in addition to anti-tuberculous drugs [4].

Tuuberculosis is a treatable disease. Patients with treated tuberculosis are expected to improve with minimal or no sequelae. Without treatment, the mortality rate of TB exceeds 50\%; [10]. Risk factors for death include old age, delay in diagnosis, extent of radiographic involvement and immunosuppression [10].

\section{CONCLUSION}

Pleural tuberculosis without parenchymal involvement may lead to diagnostic challenges. A case of 30 year old man with pleural tuberculosis misdiagnosed as pleural neoplasia based on initial clinical, laboratory and radiological evaluation was reported of which imaging played a vital role.

\section{REFERENCES}

1. Adewole, O. O., Erhabor, G. E., Ogunrombi, A. B., \& Awopeju, F. A. (2010). Prevalence and patient characteristics associated with pleural tuberculosis in Nigeria. J Infect Dev Ctries, 4(4), 213-217.

2. Khan, A. H., Sulaiman, S. A., Muttalif, A. R., Hassali, M. A., Akram, H., \& Gillani, S. W. (2013). Pleural tuberculosis and its treatment outcomes. Trop J Pharm Res, 12(4), 623-627.

3. Patel, A., \& Choudhury, S. (2013). Pleural tuberculosis presented as multiple pleural masses: An atypical presentation. Lung India, 30 (1), 54-56.
4. Padley, S. P., \& Rubens, M. B. (2003). Pulmonary infections: In Sutton D. A textbook of Radiology and Imaging. $7^{\text {th }}$ ed, London. Churchill-livingstone, $1,140-144$.

5. Antonangelo, L., Vargas, F. S., Puka, J., Seiscento, M. \& Acentio, M. M., \& Teixeira, L. R. (2012). Pleural tuberculosis Is radiological evidence of pulmonary-associated disease related to the exacerbation of the inflammatory response? Clinics (Sao Paulo). 67, (11), 1259-1263.

6. Nwonwu, E. U., Oyibo, P. G., Imo, A. O., Ndukwe, C. D., Obionu, C. N., \& Unee, C. J. (2008). Radiological features of pulmonary tuberculosis in HIV-positive and HIV-negative adult patients in South-eastern Nigeria. Afr J Resp Med, 4 (1), 2023.

7. Ikuebe, P. O., Ebuenyi, I. D., \& Ogoinja, Z. (2014). Radiological findings in pulmonary tuberculosis among HIV infected and uninfected adult patients in Yenagoa, Nigeria. IOSR J Dent Med Sci, 13(12), 80-83.

8. Kim, J. S., Shim, S. S., Kim, Y., Ryu, Y. J., \& Lee, J. H. (2014). Chest CT findings of pleural tuberculosis: differential diagnosis of pleural tuberculosis and malignant pleural dissemination. Acta Radiol. 5(9), 1063-1068.

9. Acharya, P. R., Sahoo, R., \& Baliga, P. (2008). An unusual case of pleural effusion with an abdominal mass. Indian J Tuberc, 55, 153-156.

10. Pulmonary tuberculosis: Follow up and Prognosis. www.best practice.bmj.com/.../ prognosis.html (Accessed online on $3^{\text {rd }}$ July, 2016). 